\title{
Effectiveness of XP-Endo Finisher and Passive Ultrasonic Irrigation on Intracanal Medicament Removal From Root Canals: a Systematic Review and Meta-analysis
}

Jiani Zhou

Sun Yat-Sen University

Tingjun Liu

Sun Yat-Sen University

Lihong Guo ( guolh5@mail.sysu.edu.cn )

Sun Yat-Sen University https://orcid.org/0000-0002-1241-4556

Research article

Keywords: Endodontics, intracanal medicament removal, meta-analysis, passive ultrasonic irrigation, root canal therapy, XP-Endo finisher

Posted Date: February 2nd, 2021

DOI: https://doi.org/10.21203/rs.3.rs-173700/v1

License: (c) (i) This work is licensed under a Creative Commons Attribution 4.0 International License. Read Full License 


\section{Abstract}

Background: XP-Endo finisher (XPF) and passive ultrasonic irrigation (PUI) are commonly used in intracanal medicament removal. The effectiveness of these two techniques needs to be compared, and evidence-based research should be conducted.

Methods: A comprehensive literature search was conducted in PubMed, Web of Science, Embase, Cochrane Library, and Google Scholar up to December 20th, 2020. The outcomes of the included trials were pooled into the Cochrane Collaboration's Review Manager 5.3 software. Cochrane's risk-of-bias tool 2.0 was applied to assess the risk of bias.

Results: Nine articles were included in this systematic review and processed for data extraction, and eight studies were identified for meta-analysis. In general, the use of PUI showed better medicament removal effectiveness than XPF (odds ratio [OR]: 3.09; $95 \%$ confidence interval $[\mathrm{Cl}], 1.96-4.86 ; P<0.001)$. In subgroup analysis, when anatomical areas were focused on the apical third region of the canal, PUI was also significantly more effective than XPF (OR: 3.42; 95\% Cl, 1.32-8.84; $P=0.01)$. For trials using sodium hypochlorite $(\mathrm{NaOCl})$ alone, $\mathrm{PUI}$ was also significantly more effective than XPF on intracanal medicaments removal (OR: 5.23; 95\% Cl, 2.79-9.82; $P<0.001)$. However, there was no significant difference between PUI and XPF when $\mathrm{NaOCl}$ and ethylenediaminetetraacetic acid (EDTA) were used in combination (OR: 1.51; 95\% Cl, 0.74-3.09; $P=0.26$ ). In addition, for studies whose intracanal medicament periods were two weeks, the effectiveness of PUl was statistically better than the XPF (OR: 7.73; 95\% Cl, 3.71-16.07; $P<0.001)$. Nevertheless, for trials whose intracanal medicament time was one week or over two weeks, no differences between the XPF and PUI were found (OR: 1.54; $95 \% \mathrm{Cl}, 0.74-3.22 ; P=0.25)(\mathrm{OR}: 1.42 ; 95 \% \mathrm{Cl}, 0.44-4.61 ; P=0.56)$.

Conclusions: PUI might be superior over XPF techniques for removing intracanal medicaments from artificial standardized grooves and cavities in the root canal system. The anatomical areas, irrigation protocol, and intracanal medicaments time may influence the cleaning efficacy. More large-scale and high-quality trials are warranted to validate the conclusions.

\section{Introduction}

As the research of endodontic techniques and materials has evolved greatly, the success rates of root canal treatment have improved over the years [1, 2]. It is well known that root canal treatment consists of root canal preparation, disinfection, and obturation procedures [3]. Root canal disinfection is one of the most fundamental stages in endodontic treatment because it eliminates infected dentin and nerve [4]. This procedure is critical for reducing postoperative endodontic pain and promoting longterm healing effects [5, 6]. Intracanal medicaments have been applied widely to enhance disinfection efficacy [7]. Medicaments applications contribute to outstanding cleaning effects, especially for complex anatomic areas in root canal systems, such as the apical deltas and isthmus regions [8]. Intracanal remaining medicaments should be eliminated as much as possible before root canal obturation [9]. Otherwise, the residual medicaments might adhere to the canal wall, interfere with the penetration of endodontic sealers into dentinal tubules, and increase the microleakage of obturation materials that lead to treatment failure in the long term [10-12]. Therefore, the removal of dressings placed in the root canal system before obturation is vital for achieving successful endodontic therapy.

Several studies have so far concluded that none of the applicable techniques was able to completely remove medicament substances from root canal regions [13-15]. It is urgent to compare the efficacy of various techniques in the removal of a root canal dressing, with the purpose of seeking the most effective irrigation method for clinical applications. Various techniques have been suggested for optimising intracanal medicaments removal, including syringe and needle irrigation (SNI) [16], CanalBrush (CB) [17], passive ultrasonic irrigation (PUI) [7], self-adjusting file (SAF) [18], Endo Activator (EA) [19], laser-activated irrigation (LAI) [20], and XP-endo Finisher (XPF) [21]. However, a consensus on the best method is yet to be achieved. PUI is one of the most universal and well-established irrigation methods [22]. In complex canal anatomic areas, PUI could improve the cleaning effects by cavitation and acoustic micro streaming $[14,23]$. The commonly used tip for PUI is a threaded ultrasonic file, and the instrument's size is matched to the file size based on the International Organization for Standardization [24]. The ultrasonic therapy was considered as the gold standard in the past, and it was thought to be more effective than SAF, SNI, EA, LAI, and CB in removing medicaments from the root canal system $[10,17,18,25-27]$. However, in recent years, XPF (FKG Dentaire, La Chaux de Fonds, Switzerland) was gradually applied in endodontic treatment. This novel nickel-titanium rotary finishing file could expand at body temperature with high flexibility. The characteristic also contributes to the mechanical cleaning of canal space with complex 
morphology $[28,29]$. Previous literature has analysed the functions of the XPF instrument on removing intracanal medicaments by comparing it with other techniques. Several studies reported that XPF was more effective than SNI on the removal of intracanal medicaments [30-32]. In simulated internal resorption cavities, XPF was superior to SNI, CB, and EA on the medicament removal [33]. XPF was more effective for removing calcium hydroxide residues than the XP-endo Shaper in extracted maxillary central incisors [15]. Moreover, in simulated immature root canals, the cleaning efficacy of XPF on medicaments removal was also better than SNI and EA [34].

A wide array of studies compared the efficacy of PUI with that of XPF, and their results showed clear controversy [27, 31, 34]. A systematic review by Lauritano et al. revealed that it was still unclear whether XPF could outperform PUI in terms of intracanal medicaments removal [35]. Therefore, our purpose is to quantitatively compare the efficacy of XPF with that of PUI in removing intracanal medicaments. The present study is the first meta-analysis to make a quantitative comparison between these two techniques, and it provides evidence-based results and offers clinicians a helpful guideline in endodontic therapy.

\section{Methods}

The review was carefully prepared following the Preferred Reporting Items for Systematic Reviews and Meta-Analyses (PRISMA) guidelines [36] and the 2019 Cochrane Handbook [37]. The protocol was registered in the International Prospective Register of Systematic Reviews (PROSPERO) under the number CRD42020199203.

\section{Focused question}

This systematic review and meta-analysis compared the efficacy of XPF with that of PUI on intracanal medicaments removal from root canals. The details of the focused question were as follows: 1. Participants $(P)$ : extracted human teeth with intact root canal systems. 2. Intervention (I): the use of XPF alone in removing intracanal medicaments during endodontic therapy. 3. Comparison (C): the use of PUI alone in removing intracanal medicaments during endodontic therapy. 4. Outcome (0): outcome of interest was evaluated using a scoring system described by Lee et al. [38] or van der Sluis et al. [39] according to the quantity of medicaments remained in the root canal systems.

\section{Search strategy}

Two authors (JZ and TL) independently searched for relevant literature up to December 20th, 2020 in four key databases, including PubMed, Web of Science (WoS), Embase, and Cochrane Library. In all databases mentioned above, the following search strategy was used: (passive ultrasonic activation OR passive ultrasonic irrigation OR ultrasonically activated irrigation OR passive ultrasonic agitation OR ultrasonic irrigation OR ultrasonic activation OR ultrasonic agitation OR ultrasonic therapy OR ultrasound OR PUI OR ultrasonic OR ultrasonics) AND (XP-endo Finisher file activation OR XP-endo Finisher file irrigation OR XP-endo Finisher OR XP-endo Finisher file OR XP-endo file OR Xp-endo Finisher OR XP-Finisher rotary file OR XP Endo Finisher OR Finisher file OR XP file OR XP-F OR XPF OR finisher file OR finishing file OR nickel-titanium rotary finishing file OR nickel-titanium file OR rotary file activation OR rotary finishing file OR file activation OR file irrigation). In the PubMed database, publications were sorted by 'most recent,' and the publication language was confined to 'English.' In the WoS database, the citation indexes were restricted to 'SClEXPANDED.' In the Embase database, the sources were limited to 'Embase only,' and the publication language was restricted to 'English.' In addition, the Google Scholar database was manually searched for eligible articles not indexed in the four databases mentioned above. Two authors (JZ and TL) searched each database independently and resolved disagreements by discussing their search results.

\section{Eligibility criteria}

The inclusion criteria were as follows:

1. The study was a randomised controlled trial (RCT) or controlled clinical trial (CCT).

2. The study used extracted human teeth with simulated internal resorption cavities or an artificial standardised groove in root canals.

3. XPF was involved in the experimental group(s) to remove intracanal medicaments from root canals. 
4. PUI was involved in the control group(s) to remove intracanal medicaments from root canals.

5. The outcome was evaluated using a scoring system described by Lee et al. [38] or van der Sluis et al. [39].

All specimens were split into two halves longitudinally, and the standardised grooves or resorption cavities were prepared in the root canal walls. Digital photographs of each root canal were taken before medicaments placement and after the removal of medicaments using a microscope and a digital camera. The quantity of medicaments remained in the root canal was scored: score 0 indicated that the root canal was free of medicaments; score 1 demonstrated that less than half of the root canal was covered with medicaments; score 2 indicated that more than half of the root canal was filled with medicaments; score 3 referred to a root canal entirely covered with medicaments.

Reviews, case reports, case series, letters, personal opinions, conference abstracts, book chapters, and animal studies were excluded. Moreover, relevant studies were excluded if the outcomes of interest were not extractable.

\section{Literature screening and data extraction}

All publication records were imported into EndNote X9 software, and duplications were removed. Two authors (JZ and TL) independently screened and assessed studies' titles, abstracts, and full-texts to identify eligible studies in accordance with the eligibility criteria. Irrelevant studies were excluded after the title and abstract screening. References of all eligible studies were also examined. Two authors (JZ and TL) resolved disagreements by consulting with a senior author (LG).

Data from each included study was extracted and summarised using a predefined data collection form. The relevant items were as follows: first author, year of publication, the number of teeth, intracanal medicaments time, irrigation protocol, cases, and scoring results of the XPF group and the PUI group. For dichotomous statistical analysis, the outcomes of interest were categorised, based on the scoring system, as follows: 1 . Success: medicaments were present in not more than half of a single root canal (score 0 or 1). 2. Failure: more than half of single root canal covered with medicaments (score 2 or 3 ).

\section{Risk-of-bias assessment and statistical analysis}

The Cochrane's risk-of-bias tool (RoB 2.0) was applied to assess the risk of bias arising from the following five domains: randomisation process, deviations from intended interventions, missing outcome data, measurement of the outcome, and selection of the reported result [40]. Two authors (JZ and TL) independently assessed the risk of bias in each domain following a series of signalling questions, and they evaluated the overall bias of each included trial based on the algorithm described by RoB 2.0 guidelines. If the trial had low risk in all domains, it was judged to have a low risk of overall bias. If the trial had high risk in any domain, it was judged to have a high risk of overall bias. If the trial had low risk or some concerns, with no high risk in any domain, it was considered to have some concerns overall. Disagreements between two assessors (JZ and TL) were resolved by discussion with a senior author (LG).

The outcomes of interest of the included trials were pooled into Review Manager 5.3 software (The Nordic Cochrane Centre, Copenhagen, Denmark). The odds ratio (OR) was calculated to compare the failure rate of the XPF group with that of the PUI group, and the results are reported with $95 \%$ confidence intervals (Cls). Subgroup analyses were performed based on different intracanal medicament periods, different irrigation protocols, or different root canal areas that were assessed. Statistical heterogeneity among trials was calculated by a chi-square test (significance: $p<0.10$ ) and Higgins index $(R)[41]$. The $P$ statistic indicates the diversity of variation among the eligible studies, and the diversity stems from heterogeneity rather than sampling error. If $P$ was no more than $50 \%$, we would apply the Mantel-Haenszel fixed-effects model of analysis; if $P$ was greater than $50 \%$, we would apply the random-effects model of analysis [42]. All results are displayed as ORs with $95 \%$ Cls and are shown in forest plots.

\section{Results}

\section{Literature selection}


In total, we identified 946 studies initially during the literature search (Fig. 1). After de-duplication, we browsed titles and abstracts of the remaining 770 studies, and we excluded 752 articles. Among the excluded studies, nine were reviews or meta-analysis [4351], four compared the effectiveness of PUI with XPF on removing hard-tissue debris or smear layer [52-55], three evaluated the efficacy on biofilm or bacteria removal when using the two techniques [56-58], one focused on post-operative pain of root canal treatment using the PUI versus XPF [59]. Moreover, 13 studies did not investigate the effectiveness of XPF on intracanal medicaments removal, 45 articles examined the efficacy of PUI on hard-tissue debris, smear layer, root filling remnants, bacteria, or biofilm removal. In addition, three studies only reported the efficacy of XPF instruments [15,60,61], four studies investigated the root filling remnants effects of XP-endo Finisher R instrument [62-65]. The other 670 studies focused on the fields of oral and maxillofacial medical imaging, oral and maxillofacial surgery, periodontology, prosthodontics, and other irrelated research topics. Then, a total of 18 articles underwent a full-text screening. In this phase, nine [15, 21, 66-72] studies were excluded for the following reasons: five $[15,66,69,71,72]$ lacked a comparison of medicament removal efficacy between the PUI group and XPF group, and four $[21,67,68,70]$ did not evaluate outcomes according to the scoring system described by Lee et al. or van der Sluis et al. Finally, nine articles were included in the present systematic review and processed to data extraction [27, 30-34, 73-75]. Among the nine studies included for qualitative synthesis, one was not appropriate for the quantitative synthesis, because it just presented the minimum and maximum of the scoring results in the PUI and XPF group [27]. Therefore, eight studies were identified for meta-analysis [30-34, 73-75]. References listed in all eligible studies were screened, and no additionally eligible studies were identified.

\section{Characteristics of the included studies}

The characteristics of the eligible publications are shown in Table 1. All articles are published in English. All teeth in each trial are human teeth with straight roots and a single root canal. After the removal of medicament, researchers used the same scoring system to evaluate the specimens' effects by observing digital photographs [38, 39]. A total of eight trials with 754 specimens (374 in XPF groups and 380 in PUl groups) were analysed in the quantitative synthesis stage [30-34, 73-75]. In each study, the XPF file was employed according to the manufacturer's instructions. In detail, the file tip was placed $1 \mathrm{~mm}$ short of the working length (WL), with a speed of $800 \mathrm{rpm}$ and a maximum torque of $1 \mathrm{~N} / \mathrm{cm}$. The working time was 1 minute, and the vertical movement distances were 7-8 $\mathrm{mm}$ to the full WL. In addition, in each trial included for quantitative synthesis, the ultrasonic tip in the PUI group was placed $1 \mathrm{~mm}$ short of the WL and driven by an ultrasonic device with power ranging from 5 to 9 . The working time was 1 minute. Moreover, there were two studies dividing the standardised artificial grooves into three sections: apical, coronal, and middle section [73, 74]; three trials focused on the apical third of root canals [30, 31, 75]. Furthermore, the intracanal medicament time varied among these studies. Five articles reported that medicaments were stored in the root canal for a week $[27,30,31,33,75]$, two for two weeks [73, 74], one for a month [34]. Meanwhile, a study contained three kinds of intracanal medicament periods; in detail, the medicaments were stored for 7, 21, and 90 days [32]. Moreover, a study applied both double and triple antibiotic pastes as the intracanal medications [34]. 
Table 1

The main characteristics of the included studies.

\begin{tabular}{|c|c|c|c|c|c|c|c|c|c|}
\hline \multirow{2}{*}{$\begin{array}{l}\text { Authors; } \\
\text { year }\end{array}$} & \multirow{2}{*}{$\begin{array}{l}\text { Teeth } \\
\text { number }\end{array}$} & \multirow{2}{*}{$\begin{array}{l}\text { Intracanal } \\
\text { medicament } \\
\text { time (Stored } \\
\text { at } 37^{\circ} \mathrm{C} \\
\text { with } 100 \% \\
\text { humidity) }\end{array}$} & \multirow{2}{*}{$\begin{array}{l}\text { Groups } \\
\text { (n) }\end{array}$} & \multirow[t]{2}{*}{ Irrigants } & \multirow{2}{*}{$\begin{array}{l}\text { Evaluation } \\
\text { Method }\end{array}$} & \multicolumn{2}{|c|}{ XPF group } & \multicolumn{2}{|c|}{ PUl group } \\
\hline & & & & & & Cases & $\begin{array}{l}\text { Scoring } \\
\text { result }\end{array}$ & Cases & $\begin{array}{l}\text { Scoring } \\
\text { result }\end{array}$ \\
\hline \multirow{15}{*}{$\begin{array}{l}\text { Gokturk, } \\
2016\end{array}$} & \multirow[t]{15}{*}{105} & \multirow[t]{15}{*}{ for 2 weeks } & \multirow{15}{*}{$\begin{array}{l}\text { XPF }(n \\
=15) \\
\text { PUI }(n \\
=15)\end{array}$} & \multirow[t]{15}{*}{$\mathrm{NaOCl}$} & \multirow{15}{*}{$\begin{array}{l}\text { Stereomicroscope } \\
\text { at } 20 X \\
\text { magnification }\end{array}$} & \multirow[t]{5}{*}{15} & $\begin{array}{l}\text { Coronal } \\
\text { third }\end{array}$ & \multirow[t]{5}{*}{15} & $\begin{array}{l}\text { Coronal } \\
\text { third }\end{array}$ \\
\hline & & & & & & & $\begin{array}{l}\text { score0: } \\
0\end{array}$ & & $\begin{array}{l}\text { score0: } \\
1\end{array}$ \\
\hline & & & & & & & $\begin{array}{l}\text { score1: } \\
5\end{array}$ & & $\begin{array}{l}\text { score1: } \\
9\end{array}$ \\
\hline & & & & & & & $\begin{array}{l}\text { score2: } \\
10\end{array}$ & & $\begin{array}{l}\text { score2: } \\
5\end{array}$ \\
\hline & & & & & & & $\begin{array}{l}\text { score3: } \\
0\end{array}$ & & $\begin{array}{l}\text { score3: } \\
0\end{array}$ \\
\hline & & & & & & \multirow[t]{5}{*}{15} & $\begin{array}{l}\text { Middle } \\
\text { third }\end{array}$ & \multirow[t]{5}{*}{15} & $\begin{array}{l}\text { Middle } \\
\text { third }\end{array}$ \\
\hline & & & & & & & $\begin{array}{l}\text { score0: } \\
0\end{array}$ & & $\begin{array}{l}\text { score0: } \\
0\end{array}$ \\
\hline & & & & & & & $\begin{array}{l}\text { score1: } \\
3\end{array}$ & & $\begin{array}{l}\text { score1: } \\
9\end{array}$ \\
\hline & & & & & & & $\begin{array}{l}\text { score2: } \\
11\end{array}$ & & $\begin{array}{l}\text { score2: } \\
6\end{array}$ \\
\hline & & & & & & & $\begin{array}{l}\text { score3: } \\
1\end{array}$ & & $\begin{array}{l}\text { score3: } \\
0\end{array}$ \\
\hline & & & & & & \multirow[t]{5}{*}{15} & $\begin{array}{l}\text { Apical } \\
\text { third }\end{array}$ & \multirow[t]{5}{*}{15} & $\begin{array}{l}\text { Apical } \\
\text { third }\end{array}$ \\
\hline & & & & & & & $\begin{array}{l}\text { score0: } \\
0\end{array}$ & & $\begin{array}{l}\text { score0: } \\
0\end{array}$ \\
\hline & & & & & & & $\begin{array}{l}\text { score1: } \\
1\end{array}$ & & $\begin{array}{l}\text { score1: } \\
3\end{array}$ \\
\hline & & & & & & & $\begin{array}{l}\text { score2: } \\
9\end{array}$ & & $\begin{array}{l}\text { score2: } \\
9\end{array}$ \\
\hline & & & & & & & $\begin{array}{l}\text { score3: } \\
5\end{array}$ & & $\begin{array}{l}\text { score3: } \\
3\end{array}$ \\
\hline \multirow{5}{*}{$\begin{array}{l}\text { Gokturk, } \\
2017\end{array}$} & \multirow[t]{5}{*}{105} & \multirow[t]{5}{*}{ for 2 weeks } & $\begin{array}{l}\text { XPF }(n \\
=15)\end{array}$ & \multirow[t]{5}{*}{$\mathrm{NaOCl}$} & \multirow{5}{*}{$\begin{array}{l}\text { Stereomicroscope } \\
\text { at } 20 X \\
\text { magnification }\end{array}$} & \multirow[t]{5}{*}{15} & $\begin{array}{l}\text { Coronal } \\
\text { third }\end{array}$ & \multirow[t]{5}{*}{15} & $\begin{array}{l}\text { Coronal } \\
\text { third }\end{array}$ \\
\hline & & & $\begin{array}{l}\text { PUI }(n \\
=15)\end{array}$ & & & & $\begin{array}{l}\text { score0: } \\
0\end{array}$ & & $\begin{array}{l}\text { score0: } \\
7\end{array}$ \\
\hline & & & & & & & $\begin{array}{l}\text { score1: } \\
4\end{array}$ & & $\begin{array}{l}\text { score1: } \\
5\end{array}$ \\
\hline & & & & & & & $\begin{array}{l}\text { score2: } \\
11\end{array}$ & & $\begin{array}{l}\text { score2: } \\
3\end{array}$ \\
\hline & & & & & & & $\begin{array}{l}\text { score3: } \\
0\end{array}$ & & $\begin{array}{l}\text { score3: } \\
0\end{array}$ \\
\hline
\end{tabular}




\begin{tabular}{|c|c|c|c|c|c|c|c|c|c|}
\hline \multirow{2}{*}{$\begin{array}{l}\text { Authors; } \\
\text { year }\end{array}$} & \multirow{2}{*}{$\begin{array}{l}\text { Teeth } \\
\text { number }\end{array}$} & \multirow{2}{*}{$\begin{array}{l}\text { Intracanal } \\
\text { medicament } \\
\text { time (Stored } \\
\text { at } 37^{\circ} \mathrm{C} \\
\text { with } 100 \% \\
\text { humidity) }\end{array}$} & \multirow{2}{*}{$\begin{array}{l}\text { Groups } \\
\text { (n) }\end{array}$} & \multirow[t]{2}{*}{ Irrigants } & \multirow{2}{*}{$\begin{array}{l}\text { Evaluation } \\
\text { Method }\end{array}$} & \multicolumn{2}{|c|}{ XPF group } & \multicolumn{2}{|c|}{ PUI group } \\
\hline & & & & & & Cases & $\begin{array}{l}\text { Scoring } \\
\text { result }\end{array}$ & Cases & $\begin{array}{l}\text { Scoring } \\
\text { result }\end{array}$ \\
\hline & & & & & & 15 & $\begin{array}{l}\text { Middle } \\
\text { third }\end{array}$ & 15 & $\begin{array}{l}\text { Middlle } \\
\text { third }\end{array}$ \\
\hline & & & & & & & $\begin{array}{l}\text { score0: } \\
0\end{array}$ & & $\begin{array}{l}\text { score0: } \\
7\end{array}$ \\
\hline & & & & & & & $\begin{array}{l}\text { score1: } \\
4\end{array}$ & & $\begin{array}{l}\text { score1: } \\
5\end{array}$ \\
\hline & & & & & & & $\begin{array}{l}\text { score2: } \\
9\end{array}$ & & $\begin{array}{l}\text { score2: } \\
3\end{array}$ \\
\hline & & & & & & & $\begin{array}{l}\text { score3: } \\
2\end{array}$ & & $\begin{array}{l}\text { score3: } \\
0\end{array}$ \\
\hline & & & & & & 15 & $\begin{array}{l}\text { Apical } \\
\text { third }\end{array}$ & 15 & $\begin{array}{l}\text { Apical } \\
\text { third }\end{array}$ \\
\hline & & & & & & & $\begin{array}{l}\text { score0: } \\
0\end{array}$ & & $\begin{array}{l}\text { score0: } \\
1\end{array}$ \\
\hline & & & & & & & $\begin{array}{l}\text { score1: } \\
0\end{array}$ & & $\begin{array}{l}\text { score1: } \\
7\end{array}$ \\
\hline & & & & & & & $\begin{array}{l}\text { score2: } \\
7\end{array}$ & & $\begin{array}{l}\text { score2: } \\
7\end{array}$ \\
\hline & & & & & & & $\begin{array}{l}\text { score3: } \\
8\end{array}$ & & $\begin{array}{l}\text { score3: } \\
0\end{array}$ \\
\hline \multirow{4}{*}{$\begin{array}{l}\text { Keskin, } \\
2017\end{array}$} & 100 & for 1 week & $\begin{array}{l}\text { XPF }(n \\
=18)\end{array}$ & $\begin{array}{l}\mathrm{NaOCl} \\
+ \text { EDTA }\end{array}$ & $\begin{array}{l}\text { Stereomicroscope } \\
\text { at } 20 \mathrm{X}\end{array}$ & 36 & $\begin{array}{l}\text { score0: } \\
9\end{array}$ & 36 & $\begin{array}{l}\text { score0: } \\
16\end{array}$ \\
\hline & & & $\begin{array}{l}\text { PUI }(n \\
=18)\end{array}$ & & & & $\begin{array}{l}\text { score1: } \\
12\end{array}$ & & $\begin{array}{l}\text { score1: } \\
11\end{array}$ \\
\hline & & & & & & & $\begin{array}{l}\text { score2: } \\
11\end{array}$ & & $\begin{array}{l}\text { score2: } \\
5\end{array}$ \\
\hline & & & & & & & $\begin{array}{l}\text { score3: } \\
4\end{array}$ & & $\begin{array}{l}\text { score3: } \\
4\end{array}$ \\
\hline \multirow{5}{*}{$\begin{array}{l}\text { Uygun, } \\
2017\end{array}$} & 32 & for 1 week & $\begin{array}{l}\text { XPF }(n \\
=8)\end{array}$ & EDTA & $\begin{array}{l}\text { Stereomicroscope } \\
\text { at } 25 \mathrm{X}\end{array}$ & 16 & $\begin{array}{l}\text { Apical } \\
\text { third }\end{array}$ & 16 & $\begin{array}{l}\text { Apical } \\
\text { third }\end{array}$ \\
\hline & & & $\begin{array}{l}\text { PUI }(n \\
=8)\end{array}$ & & & & $\begin{array}{l}\text { score0: } \\
12\end{array}$ & & $\begin{array}{l}\text { score0: } \\
14\end{array}$ \\
\hline & & & & & & & $\begin{array}{l}\text { score1: } \\
3\end{array}$ & & $\begin{array}{l}\text { score1: } \\
2\end{array}$ \\
\hline & & & & & & & $\begin{array}{l}\text { score2: } \\
1\end{array}$ & & $\begin{array}{l}\text { score2: } \\
0\end{array}$ \\
\hline & & & & & & & $\begin{array}{l}\text { score3: } \\
0\end{array}$ & & $\begin{array}{l}\text { score3: } \\
0\end{array}$ \\
\hline
\end{tabular}




\begin{tabular}{|c|c|c|c|c|c|c|c|c|c|}
\hline \multirow{2}{*}{$\begin{array}{l}\text { Authors; } \\
\text { year }\end{array}$} & \multirow{2}{*}{$\begin{array}{l}\text { Teeth } \\
\text { number }\end{array}$} & \multirow{2}{*}{$\begin{array}{l}\text { Intracanal } \\
\text { medicament } \\
\text { time (Stored } \\
\text { at } 37^{\circ} \mathrm{C} \\
\text { with } 100 \% \\
\text { humidity) }\end{array}$} & \multirow{2}{*}{$\begin{array}{l}\text { Groups } \\
\text { (n) }\end{array}$} & \multirow[t]{2}{*}{ Irrigants } & \multirow{2}{*}{$\begin{array}{l}\text { Evaluation } \\
\text { Method }\end{array}$} & \multicolumn{2}{|c|}{ XPF group } & \multicolumn{2}{|c|}{ PUl group } \\
\hline & & & & & & Cases & $\begin{array}{l}\text { Scoring } \\
\text { result }\end{array}$ & Cases & $\begin{array}{l}\text { Scoring } \\
\text { result }\end{array}$ \\
\hline \multirow{5}{*}{$\begin{array}{l}\text { Wigler, } \\
2017\end{array}$} & \multirow[t]{5}{*}{68} & \multirow[t]{5}{*}{ for 1 week } & $\begin{array}{l}\text { XPF }(n \\
=20)\end{array}$ & \multirow[t]{5}{*}{$\mathrm{NaOCl}$} & \multirow[t]{5}{*}{$\begin{array}{l}\text { Microscope at } 24 \\
\mathrm{X} \text { magnification }\end{array}$} & \multirow[t]{5}{*}{20} & $\begin{array}{l}\text { Apical } \\
\text { third }\end{array}$ & \multirow[t]{5}{*}{20} & $\begin{array}{l}\text { Apical } \\
\text { third }\end{array}$ \\
\hline & & & $\begin{array}{l}\text { PUI }(n \\
=20)\end{array}$ & & & & $\begin{array}{l}\text { score0: } \\
0\end{array}$ & & $\begin{array}{l}\text { score0: } \\
0\end{array}$ \\
\hline & & & & & & & $\begin{array}{l}\text { score1: } \\
2\end{array}$ & & $\begin{array}{l}\text { score1: } \\
3\end{array}$ \\
\hline & & & & & & & $\begin{array}{l}\text { score2: } \\
14\end{array}$ & & $\begin{array}{l}\text { score2: } \\
14\end{array}$ \\
\hline & & & & & & & $\begin{array}{l}\text { score3: } \\
4\end{array}$ & & $\begin{array}{l}\text { score3: } \\
3\end{array}$ \\
\hline \multirow{15}{*}{$\begin{array}{l}\text { Keskin, } \\
2018\end{array}$} & \multirow[t]{15}{*}{190} & \multirow[t]{15}{*}{$\begin{array}{l}\text { for } 7,21 \text { or } \\
90 \text { days }\end{array}$} & \multirow{15}{*}{$\begin{array}{l}\text { XPF }(n \\
=20) \\
\text { PUI }(n \\
=20)\end{array}$} & \multirow{15}{*}{$\begin{array}{l}\mathrm{NaOCl} \\
+\mathrm{EDTA}\end{array}$} & \multirow{15}{*}{$\begin{array}{l}\text { Stereomicroscope } \\
\text { at } 30 \mathrm{X} \\
\text { magnification }\end{array}$} & \multirow[t]{5}{*}{34} & 7 days & \multirow[t]{5}{*}{40} & 7 days \\
\hline & & & & & & & $\begin{array}{l}\text { score0: } \\
19\end{array}$ & & $\begin{array}{l}\text { score0: } \\
20\end{array}$ \\
\hline & & & & & & & $\begin{array}{l}\text { score1: } \\
15\end{array}$ & & $\begin{array}{l}\text { score1: } \\
18\end{array}$ \\
\hline & & & & & & & $\begin{array}{l}\text { score2: } \\
0\end{array}$ & & $\begin{array}{l}\text { score2: } \\
2\end{array}$ \\
\hline & & & & & & & $\begin{array}{l}\text { score3: } \\
0\end{array}$ & & $\begin{array}{l}\text { score3: } \\
0\end{array}$ \\
\hline & & & & & & \multirow[t]{5}{*}{40} & $\begin{array}{l}21 \\
\text { days }\end{array}$ & \multirow[t]{5}{*}{40} & $\begin{array}{l}21 \\
\text { days }\end{array}$ \\
\hline & & & & & & & $\begin{array}{l}\text { score0: } \\
11\end{array}$ & & $\begin{array}{l}\text { score0: } \\
17\end{array}$ \\
\hline & & & & & & & $\begin{array}{l}\text { score1: } \\
28\end{array}$ & & $\begin{array}{l}\text { score1: } \\
22\end{array}$ \\
\hline & & & & & & & $\begin{array}{l}\text { score2: } \\
1\end{array}$ & & $\begin{array}{l}\text { score2: } \\
1\end{array}$ \\
\hline & & & & & & & $\begin{array}{l}\text { score3: } \\
0\end{array}$ & & $\begin{array}{l}\text { score3: } \\
0\end{array}$ \\
\hline & & & & & & \multirow[t]{5}{*}{40} & $\begin{array}{l}90 \\
\text { days }\end{array}$ & \multirow[t]{5}{*}{40} & $\begin{array}{l}90 \\
\text { days }\end{array}$ \\
\hline & & & & & & & $\begin{array}{l}\text { score0: } \\
8\end{array}$ & & $\begin{array}{l}\text { score0: } \\
18\end{array}$ \\
\hline & & & & & & & $\begin{array}{l}\text { score1: } \\
32\end{array}$ & & $\begin{array}{l}\text { score } 1: \\
22\end{array}$ \\
\hline & & & & & & & $\begin{array}{l}\text { score2: } \\
0\end{array}$ & & $\begin{array}{l}\text { score2: } \\
0\end{array}$ \\
\hline & & & & & & & $\begin{array}{l}\text { score3: } \\
0\end{array}$ & & $\begin{array}{l}\text { score3: } \\
0\end{array}$ \\
\hline
\end{tabular}




\begin{tabular}{|c|c|c|c|c|c|c|c|c|c|}
\hline \multirow{2}{*}{$\begin{array}{l}\text { Authors; } \\
\text { year }\end{array}$} & \multirow{2}{*}{$\begin{array}{l}\text { Teeth } \\
\text { number }\end{array}$} & \multirow{2}{*}{$\begin{array}{l}\text { Intracanal } \\
\text { medicament } \\
\text { time (Stored } \\
\text { at } 37{ }^{\circ} \mathrm{C} \\
\text { with } 100 \% \\
\text { humidity) }\end{array}$} & \multirow{2}{*}{$\begin{array}{l}\text { Groups } \\
\text { (n) }\end{array}$} & \multirow[t]{2}{*}{ Irrigants } & \multirow{2}{*}{$\begin{array}{l}\text { Evaluation } \\
\text { Method }\end{array}$} & \multicolumn{2}{|c|}{ XPF group } & \multicolumn{2}{|c|}{ PUl group } \\
\hline & & & & & & Cases & $\begin{array}{l}\text { Scoring } \\
\text { result }\end{array}$ & Cases & $\begin{array}{l}\text { Scoring } \\
\text { result }\end{array}$ \\
\hline \multirow{5}{*}{$\begin{array}{l}\text { Kfir, } \\
2018\end{array}$} & \multirow[t]{5}{*}{80} & \multirow[t]{5}{*}{ for 1 week } & $\begin{array}{l}\text { XPF }(n \\
=18)\end{array}$ & \multirow[t]{5}{*}{$\mathrm{NaOCl}$} & \multirow[t]{5}{*}{$\begin{array}{l}\text { Microscope at } 24 \\
\text { X magnification }\end{array}$} & \multirow[t]{5}{*}{18} & $\begin{array}{l}\text { Apical } \\
\text { third }\end{array}$ & \multirow[t]{5}{*}{18} & $\begin{array}{l}\text { Apical } \\
\text { third }\end{array}$ \\
\hline & & & $\begin{array}{l}\text { PUI }(n \\
=18)\end{array}$ & & & & $\begin{array}{l}\text { score } 0: \\
0\end{array}$ & & $\begin{array}{l}\text { score0: } \\
0\end{array}$ \\
\hline & & & & & & & $\begin{array}{l}\text { score } 1: \\
2\end{array}$ & & $\begin{array}{l}\text { score1: } \\
2\end{array}$ \\
\hline & & & & & & & $\begin{array}{l}\text { score2: } \\
13\end{array}$ & & $\begin{array}{l}\text { score2: } \\
13\end{array}$ \\
\hline & & & & & & & $\begin{array}{l}\text { score3: } \\
3\end{array}$ & & $\begin{array}{l}\text { score3: } \\
3\end{array}$ \\
\hline \multirow{2}{*}{$\begin{array}{l}\text { Donnermeyer, } \\
2019\end{array}$} & \multirow[t]{2}{*}{90} & \multirow[t]{2}{*}{ for 1 week } & $\begin{array}{l}\text { XPF }(n \\
=20)\end{array}$ & \multirow[t]{2}{*}{$\mathrm{NaOCl}$} & \multirow{2}{*}{$\begin{array}{l}\text { Laser scanning } \\
\text { microscope at } 10 \\
\mathrm{X} \text { magnification }\end{array}$} & \multirow[t]{2}{*}{20} & \multirow[t]{2}{*}{ NA } & \multirow[t]{2}{*}{20} & \multirow[t]{2}{*}{ NA } \\
\hline & & & $\begin{array}{l}\text { PUI }(\mathrm{n} \\
=20)\end{array}$ & & & & & & \\
\hline \multirow[t]{9}{*}{$\begin{array}{l}\text { Sarıyılmaz, } \\
2019\end{array}$} & \multirow[t]{9}{*}{180} & \multirow[t]{9}{*}{ for 1 month } & $\begin{array}{l}\text { XPF (n } \\
=20)\end{array}$ & \multirow{9}{*}{$\begin{array}{l}\mathrm{NaOCl} \\
+\mathrm{EDTA}\end{array}$} & \multirow{9}{*}{$\begin{array}{l}\text { Stereomicroscope } \\
\text { at } 10 x \\
\text { magnification }\end{array}$} & \multirow[t]{4}{*}{40} & $\begin{array}{l}\text { DAP } \\
\text { score0: } \\
20\end{array}$ & \multirow[t]{4}{*}{40} & \multirow{4}{*}{$\begin{array}{l}\text { DAP } \\
\text { score0: } \\
13 \\
\text { score1: } \\
26 \\
\text { score2: } \\
1 \\
\text { score3: } \\
0\end{array}$} \\
\hline & & & $\begin{array}{l}\text { PUI }(\mathrm{n} \\
=20)\end{array}$ & & & & $\begin{array}{l}\text { scoreu: } \\
20 \\
\text { score1: } \\
16\end{array}$ & & \\
\hline & & & & & & & $\begin{array}{l}\text { score2: } \\
4\end{array}$ & & \\
\hline & & & & & & & $\begin{array}{l}\text { score3: } \\
0\end{array}$ & & \\
\hline & & & & & & \multirow[t]{5}{*}{40} & TAP & \multirow[t]{5}{*}{40} & TAP \\
\hline & & & & & & & $\begin{array}{l}\text { score0: } \\
23\end{array}$ & & $\begin{array}{l}\text { score0: } \\
19\end{array}$ \\
\hline & & & & & & & $\begin{array}{l}\text { score1: } \\
15\end{array}$ & & $\begin{array}{l}\text { score1: } \\
18\end{array}$ \\
\hline & & & & & & & $\begin{array}{l}\text { score2: } \\
2\end{array}$ & & $\begin{array}{l}\text { score2: } \\
3\end{array}$ \\
\hline & & & & & & & $\begin{array}{l}\text { score3: } \\
0\end{array}$ & & $\begin{array}{l}\text { score3: } \\
0\end{array}$ \\
\hline
\end{tabular}

\section{Risk of bias assessment}

The risk of bias analysis is shown in Fig. 2 and presented in detail in Fig. 3. All nine included studies were judged to raise some concerns overall. Although all the included trials mentioned the term 'randomly' to describe the method of dividing the samples, specific randomisation methods were not mentioned in eight trials. Only one manuscript described that it carried out a random sequence generated by flipping coins [30]. Meanwhile, none of the included literature performed allocation concealment. Therefore, nine studies (100\%) showed some concerns about randomisation process bias. There were no deviations from intended interventions, missing outcome data, or reporting bias in all the eligible trials. Concerning the bias of outcome 
measurement, all the included studies evaluated the outcomes using the four-grade scoring system described by Lee et al. [38] or van der Sluis et al. [39]. In addition, in all included studies, the amount of the remaining medicament was independently scored by two calibrated and blinded doctors. Consequently, all eligible studies were deemed to have a low risk of bias in the measurement of the outcome.

\section{Quantitative synthesis}

Outcome data from a total of 754 teeth or root halves were collected to calculate the overall removal efficacy between the XPF and PUI group. The failure rate of removal intracanal medicaments was 34.8\% (130/374) in the XPF group and 22.4\% (85/380) in the PUI group. Since the heterogeneity was low across the trials, we applied a fixed-effects model $\left(\chi^{2}=15.94\right.$, degrees of freedom $=13, P=0.25, R=18 \%$; Fig. 4). The overall effect showed that PUI had a greater medicaments removal effectiveness than XPF (OR: $3.09 ; 95 \% \mathrm{Cl}, 1.96-4.86 ; P<0.001 ;$ Fig. 4). Additionally, a subgroup analysis also indicated that PUI was significantly more effective than XPF in terms of removing medicaments in the apical third (OR: 3.42; 95\% Cl, 1.32-8.84; $P=0.01$; Fig. 5A). Furthermore, we carried out a subgroup analysis based on irrigants protocols. For trials using $\mathrm{NaOCl}$ alone, data showed a significantly better intracanal medicaments removal rate in the PUI group than XPF (OR: 5.23; $95 \% \mathrm{Cl}, 2.79-9.82 ; P<0.001$; Fig. 5B). However, for studies combining $\mathrm{NaOCl}$ and EDTA as canal irrigants, the forest plot did not demonstrate significant differences between PUI and XPF group (OR: 1.51; $95 \% \mathrm{Cl}, 0.74-3.09 ; P=0.26$; Fig. 5B). Nonetheless, concerning the intracanal cleaning effectiveness, a better outcome trend was found in PUI than XPF group. There was no heterogeneity revealed among these data $\left(X^{2}=3.70\right.$, degrees of freedom $=4, P=0.45, R=0 \%$; Fig. $\left.5 B\right)$. Moreover, the subgroup analysis based on intracanal medicament time was conducted. For trials whose intracanal medicament time was two weeks, the result showed that medicaments removal effectiveness of PUI was statistically better than that of XPF (OR: 7.73; $95 \% \mathrm{Cl}, 3.71-16.07 ; P<0.001$; Fig. 5C). Nevertheless, for trials whose intracanal medicament time was one week, no differences between the XPF and PUI group were found (OR: 1.54; 95\% Cl, 0.74-3.22; $P=0.25 ; \mathrm{Fig} .5 \mathrm{C}$ ). For trials whose intracanal medicament time was over two weeks, no differences were observed, either (OR: $1.42 ; 95 \% \mathrm{Cl}, 0.44-4.61 ; P=0.56$; Fig. 5C).

\section{Discussion}

One of the main goals of root canal disinfection is to eliminate microorganisms [76]. However, it is feared that the solubility of residual medicaments in tissue fluids might facilitate bacterial proliferation, because remnants might prevent sealers adaption and leave voids in the filling dentine interface $[7,77]$. Several studies have focused on improving the removal of intracanal medicaments during endodontic treatment process, but none of the activation regimens can render the root canals completely free of dressing material $[13,66,78]$. Numerous studies have suggested the superiority of XPF and PUI over other instruments on intracanal medicaments removal $[30,79]$. However, a consensus on which approach is better remains to be reached.

\section{Summary of the main results}

This is the first meta-analysis to quantitatively analyse the effect of XPF versus PUI on cleaning residual medicaments in the root canal system. In the present meta-analysis, results suggested that, in general, protocols using PUI were more effective than protocols using XPF in removing medicaments from single straight root canals. The anatomical areas, irrigation protocol, and intracanal medicaments time may influence the cleaning efficacy. Concerning anatomy area on the apical third of root canals, PUI operated superiorly over XPF. In addition, XPF had higher requirements for flushing agents than PUI. XPF might require combining $\mathrm{NaOCl}$ and EDTA as the irrigants for performing more effectively.

\section{Overall completeness and applicability of evidence}

In the present meta-analysis, a comprehensive literature search was conducted using multiple databases. In addition to the four key databases, the Google Scholar database and the reference lists of included trials were searched manually. Meanwhile, all included trials were performed by experienced operators in endodontology institutions in college or specialised clinical centres. When trials were operated in different clinical settings, results might change to some extent. In addition, the irrigation protocol of the XPF group was employed according to the manufacturer's instructions. The PUI group was also performed utilising an ultrasonic tip with a specific placed site, total activation time, and power range. The efficacies of XPF and PUI on medicament removal might be affected by different operation protocols.

Page $10 / 22$ 


\section{Potential bias in the review process}

Seven of the eight included studies did not clarify the randomisation methods in detail, and none of the included trials performed allocation concealment. In addition, considering that ultrasonic file tips have various appearances with XPF file, it was impractical to blind the operators when using instruments in XPF and PUI groups. Lacking the clarification of randomisation and allocation concealment may exaggerate the estimates of intervention effect and contribute to the bias of randomisation process [40]. A high-quality RCT should properly carry out and elaborate on the process of randomisation and allocation concealment, and future trials related to the research topic are encouraged to conduct the process more rigorously. In all included articles, bias arising from intended intervention deviations, measurement of the outcome, missing outcome data, and reporting bias were regarded as 'low risk.' All eligible trials reported that evaluators were blinded to group allocation and evaluated the results using the same fourgrade scoring system. There was no clear bias arising from the measurement of the outcome. Moreover, bias due to missing outcome data was not observed in the include studies. Regarding the reporting bias, the results in all eligible studies were reported in accordance with the pre-specified outcome assessment criteria. As a result, the reporting bias was avoided in all trials. Overall, bias in all domain might be minimised in future RCTs.

\section{Agreements and disagreements with other studies or reviews}

In recent years, studies on the cleaning effects of instruments in the root canal systems attract more and more attention [80-82]. However, the experimental conditions were difficult to standardise in the complex curved canals, because the range of length, radius, curvature, and isthmus of canals could affect the experimental results [83]. The clinical trials that compared the cleaning capacities of PUI and XPF in the curved canals were scarce and not related to our research topic [58]. Merely four studies focused on the effectiveness of calcium hydroxide removal using PUI in the curved canals, and they did not compare it with XPF [78, 8385]. Moreover, two trials researched the cleaning efficacy of XPF instrument, but they did not focus on the intracanal medicaments removal effects $[86,87]$. So far, the intracanal medicaments removal efficacy of PUI and XPF has not been compared in curved root canals, hence all trials included in the meta-analysis were conducted only in the single straight root canals.

The present quantitative synthesis illustrated a better effectiveness of PUI than XPF on intracanal medicaments removal in single straight root canals, which can be explained by the following reasons. The file size, working mechanism, and tip insertion position of PUI could benefit its effects in single straight root canals. First, tip size of the PUI file is matched to the intracanal diameters, and the final preparation size of the physiological foramen. Before the process of root canal disinfection, the single straight root canals were usually prepared up to size $40 / 0.04$, which reached a balance among cleaning effectiveness, anatomical enlargement, and the apical accident risk [33, 75, 80]. However, XPF is a size 25 , and nontapered instrument, thereby the size of XPF might be less matched to the foramen size than PUI [29]. Second, the working mechanism of the PUI depends on higher velocity and irrigant stream, along with a thermal effect and the transmission of energy $[88,89]$. It contributes to the cleaning effects on the large straight canal space by flowing in the apical-to-coronal direction [90, 91]. A study published by Sabins et al. found that a working time of 30 seconds was sufficient for the PUI to exert a higher cleaning effect than SNI [92]. However, in the large canal space, one minute was probably not sufficient for the XPF to remove intracanal medicaments efficiently [30, 75]. Meantime, Kfir et al. and Wigler et al. also worried that the contact time between the XPF file and the groove in straight root canals was too short [30,75]. Third, the situation of the tip placement would impact the irrigation effects in endodontic treatment [93]. Uzunoglu et al. reported that PUI might decrease the amount of irrigant extruded through the apex [94]. In teeth with open apex, Peeters et al. also revealed that use of PUI during final irrigation procedures barely resulted in apical extrusion of $\mathrm{NaOCl}$ in endodontic therapy [95]. One explanation is that with the insertion depth of the ultrasonic tip becoming deeper, the amounts of debris and irrigants extrusion would also increase [96]. Therefore, from the standpoint of efficiency and safety, the use of PUI was better than XPF in intracanal medicaments removal in single straight root canals.

However, the cleaning effectiveness of PUI might be reduced in the complex curved root canals. Amato et al. compared the ultrasonic action efficiency between straight and curved root canals, founding that the dental debris removal efficacy of PUI could be decreased in curved root canals [97]. One possible explanation was that touching the curved root canal wall would reduce the action of ultrasonic inevitably [97]. Compared with the PUI files, XPF could expand more flexibly and have better fracture resistance, making it better adapt to the irregular anatomy of curved canals [87]. In detail, the XPF could change to a unique

Page $11 / 22$ 
spoon shape and adapt three-dimensionally to the root canal morphology at body temperature [90]. Meanwhile, the file has good resistance to fatigue and high stress, which is of particular importance for irrigation in curved root canals. Vaz-Garcia et al. concluded that XPF instruments performed better when compared its cyclic fatigue with that of the other anatomic finishing file, XP-Clean instruments [98]. However, ultrasonic tips might fracture during the endodontic shaping process [99]. In addition, Song et al. suggested that pre-curved files removed calcium hydroxide more effectively than the none-pre-curved files in curved root canals [84]. However, during the PUI procedure, pre-curving the ultrasonic file to fully adapt to the curved root canals is challenging. The tip of the PUI file unable to fully extend into the apical position because straight-line access is difficult to build [100]. Therefore, when the tip of the XPF file showed a unique spoon-shape and flexibly extended into the apical complex region in the curved root canal, the effects of intracanal medicaments removal might be higher than using the PUI file. [99]. Further studies are encouraged to investigate the efficacy of instruments on intracanal remnants removal in the curved root canal regions.

In addition, as the subgroup analyses showed, in teeth with single straight root canals, anatomical areas, irrigant protocols, and intracanal medicament time might influence the overall intracanal medicaments removal effectiveness.

Consistent with some previous studies [73, 74], PUI were superior to XPF for removing medicaments from the apical third regions of single straight root canals. A systematic review published by Yaylali et al. showed that PUI was superior over SNI and EA for removing calcium hydroxide from the root canal apical third area [101]. It is more difficult for the irrigation techniques to fully contact with the canal wall because the apical third regions have more lateral canals, apical ramifications, and isthmus than the coronal and middle third regions[102]. In addition to the complex anatomical factors, the phenomenon of vapor lock also prevented the irrigant solutions to penetrate into apical third regions [103]. PUI was effective in eliminating vapor lock during endodontic irrigation in the apical third of the root canal region [104]. Donnermeyer et al. also reported that PUI was significantly better than XPF in the removal of medicaments from the apical region [27].

In addition, the meta-analysis suggested that PUl performed more effectively than XPF when $\mathrm{NaOCl}$ was used as the only flushing agent. $\mathrm{NaOCl}$ and EDTA have been the most commonly used irrigating solutions, with the function of dissolving organic substances, killing microbes, and cooling files $[105,106]$. Lee et al. reported that the combined use of ultrasound and $\mathrm{NaOCl}$ led to synergistic effects on reducing bacteria on steel and iceberg lettuce [107]. Therefore, using $\mathrm{NaOCl}$ as the irrigant might enhance the working efficacy of PUI. However, when $\mathrm{NaOCl}$ and EDTA were used in combination, the cleaning efficacy of the PUI and XPF was similar. Azimian et al. assessed the efficacy of XPF on the removal of smear layer and residual debris [108]. They suggested that the synergistic effect of XPF and EDTA was beneficial for better root canal cleaning effects [108]. With the assistance of EDTA, XPF could achieve similar cleaning effectiveness with PUI on the removal of intracanal medicaments. In addition, the concentrations of irrigants and operation temperature might also influence the cleaning effectiveness, but the specific mechanisms are still unclear [109].

\section{Strengths and limitations}

The present systematic review and meta-analysis had several advantages. First, two authors searched key databases independently using an adequate searching strategy. It provided a precise screen range and improved the possibility of generalising the outcomes [110]. Second, the detailed inclusion and exclusion criteria were defined beforehand. We focused on the process of intracanal medicaments removal and excluded articles comparing the efficacy of removing other substances in endodontic treatment, such as smear layer, bacteria, and organic tissue. It should be noted that these listed substances were cleaned in different periods of endodontic therapy. The files in different preparation or disinfection process might play different key roles [90]. Third, only RCTs and CCTs, which are regarded as the highest level of evidence, were included in our quantitative analysis [111]. Moreover, prior protocol registration and subgroup analysis were also the strengths of the current meta-analysis $[112,113]$. Different root canal area, irrigation protocols, or intracanal medicament periods are potential factors affecting the heterogeneity. To our knowledge, the meta-analysis is the first study to quantitatively compare the effectiveness of XPF with that of PUI techniques on intracanal medicaments removal.

Several limitations exist in the present meta-analysis. First, language was limited to English during the literature screening process, and this might increase the possibility of reporting bias. When compared to non-English-language journals, Englishlanguage journals are more likely to publish positive results [91]. Second, because the case number of some studies was small,

Page 12/22 
the statistical power might be reduced. Third, in most of the included trials, the description of RCT was too simple, and it would influence the quality of studies. Accordingly, improving the quality of related RCTs is advocated.

\section{Conclusions}

The present systematic review and meta-analysis indicated that neither XPF nor PUI could completely remove medicament substances from root regions. In addition, PUI might be superior over XPF protocols for removing medicaments from the single straight root canal area. The review provided evidence-based results and might build a useful guideline for clinical application. Further studies should seek an irrigation device that could render the root canal free of medication completely. What is more, to further assess the efficacy of XPF and PUI techniques in intracanal medicament removal, more large-scale and high-quality RCTs will be warranted in the future.

\section{Abbreviations}

XPF: XP-Endo finisher; PUI: passive ultrasonic irrigation; OR: odds ratio; Cl: confidence interval; NaOCl: sodium hypochlorite; EDTA: ethylenediaminetetraacetic acid; SNI: syringe and needle irrigation; CB: Canal Brush; SAF: self-adjusting file; EA: Endo Activator; LAI: laser-activated irrigation; PRISMA: Preferred Reporting Items for Systematic Reviews and Meta-Analyses; PROSPERO: International Prospective Register of Systematic Reviews; WoS: Web of Science; RCT: randomised controlled trial; CCT: controlled clinical trial; RoB: risk-of-bias; WL: working length

\section{Declarations}

\section{Ethics approval and consent to participate}

Not applicable.

\section{Consent for publication}

Not applicable.

\section{Availability of data and materials}

All data generated or analysed during this study are included in this published article.

\section{Competing interests}

The authors declare that they have no competing interests.

\section{Funding}

This work was funded by a grant from the National Natural Science Foundation of China to LHG (funding number: 81670982).

\section{Authors' contributions}

JZ and TL participated in searching literature, data extractions, statistical analysis, and drafting the manuscript. LG designed the study, participated in data extractions, performed the statistical analysis, and revised the manuscript. All authors carefully revise and approved the final manuscript.

\section{Acknowledgements}

Not applicable.

\section{References}


1. Chybowski EA, Glickman GN, Patel Y, Fleury A, Solomon E, He J. Clinical Outcome of Non-Surgical Root Canal Treatment Using a Single-cone Technique with Endosequence Bioceramic Sealer: A Retrospective Analysis. J Endod. 2018;44(6):941945.

2. Llena C, Nicolescu T, Perez S, Gonzalez de Pereda S, Gonzalez A, Alarcon I, Monzo A, Sanz JL, Melo M, Forner L. Outcome of Root Canal Treatments Provided by Endodontic Postgraduate Students. A Retrospective Study. J Clin Med. 2020;9(6).

3. Öter B, Topçuog Lu N, Tank MK, Çehreli SB. Evaluation of Antibacterial Efficiency of Different Root Canal Disinfection Techniques in Primary Teeth. Photomed Laser Surg. 2018;36(4):179-184.

4. Gazzaneo I, Vieira GCS, Pérez AR, Alves FRF, Gonçalves LS, Mdala I, Siqueira JF, Jr., Rôças IN. Root Canal Disinfection by Single- and Multiple-instrument Systems: Effects of Sodium Hypochlorite Volume, Concentration, and Retention Time. J Endod. 2019;45(6):736-741.

5. Singh RD, Khatter R, Bal RK, Bal CS. Intracanal medications versus placebo in reducing postoperative endodontic pain--a double-blind randomized clinical trial. Braz Dent J. 2013;24(1):25-29.

6. Gama TG, de Oliveira JC, Abad EC, Rôças IN, Siqueira JF, Jr. Postoperative pain following the use of two different intracanal medications. Clin Oral Investig. 2008;12(4):325-330.

7. Silva LJ, Pessoa OF, Teixeira MB, Gouveia CH, Braga RR. Micro-CT evaluation of calcium hydroxide removal through passive ultrasonic irrigation associated with or without an additional instrument. Int Endod J. 2015;48(8):768-773.

8. Valverde ME, Baca P, Ceballos L, Fuentes MV, Ruiz-Linares M, Ferrer-Luque CM. Antibacterial efficacy of several intracanal medicaments for endodontic therapy. Dent Mater J. 2017;36(3):319-324.

9. de Freitas JV, Ebert J, Mazzi-Chaves JF, de Sousa-Neto MD, Lohbauer U, Baratto-Filho F. Do Contaminating Substances Influence the Rheological Properties of Root Canal Sealers? J Endod. 2020;46(2):258-263.

10. Capar ID, Ozcan E, Arslan H, Ertas H, Aydinbelge HA. Effect of different final irrigation methods on the removal of calcium hydroxide from an artificial standardized groove in the apical third of root canals. J Endod. 2014;40(3):451-454.

11. Keles A, Ahmetoglu F, Uzun I. Quality of different gutta-percha techniques when filling experimental internal resorptive cavities: a micro-computed tomography study. Aust Endod J. 2014;40(3):131-135.

12. Barbizam JV, Trope M, Teixeira EC, Tanomaru-Filho M, Teixeira FB. Effect of calcium hydroxide intracanal dressing on the bond strength of a resin-based endodontic sealer. Braz Dent J. 2008;19(3):224-227.

13. Camargo CH, Leal FM, Silva GO, de Oliveira TR, Madureira PG, Camargo SE. Efficacy of different techniques for removal of calcium hydroxide-chlorhexidine paste from root canals. Gen Dent. 2016;64(2):e9-12.

14. Kirar DS, Jain P, Patni P. Comparison of different irrigation and agitation methods for the removal of two types of calcium hydroxide medicaments from the root canal wall: an in-vitro study. Clujul Med. 2017;90(3):327-332.

15. Denna J, Shafie LA, Alsofi L, Al-Habib M, AlShwaimi E. Efficacy of the Rotary Instrument XP-Endo Finisher in the Removal of Calcium Hydroxide Intracanal Medicament in Combination with Different Irrigation Techniques: A Microtomographic Study. Materials (Basel). 2020;13(10).

16. Akman M, Akbulut MB, Aydınbelge HA, Belli S. Comparison of different irrigation activation regimens and conventional irrigation techniques for the removal of modified triple antibiotic paste from root canals. J Endod. 2015;41(5):720-724.

17. Zorzin J, Wießner J, Wießner T, Lohbauer U, Petschelt A, Ebert J. Removal of Radioactively Marked Calcium Hydroxide from the Root Canal: Influence of Volume of Irrigation and Activation. J Endod. 2016;42(4):637-640.

18. Ahmetoğlu F, Şımşek N, Keleş A, Ocak MS, Er K. Effıcacy of self-adjusting file and passive ultrasonic irrigation on removing calcium hydroxide from root canals. Dent Mater J. 2013;32(6):1005-1010.

19. Acharya N, Poudel D, Chakradhar A. A Comparative Evaluation of Removal of Intracanal Calcium Hydroxide with Endoactivator System and Mechanical Instrumentation with K File, Using Two Irrigating Solutions: an in Vitro Study. Kathmandu Univ Med J (KUMJ). 2018;16(61):74-77.

20. Kustarci A, Er K, Siso SH, Aydin H, Harorli H, Arslan D, Kirmali O. Efficacy of Laser-Activated Irrigants in Calcium Hydroxide Removal from the Artificial Grooves in Root Canals: An Ex Vivo Study. Photomedicine and Laser Surgery. 2016;34(5):205-210.

21. Hamdan R, Michetti J, Pinchon D, Diemer F, Georgelin-Gurgel M. The XP-Endo Finisher for the removal of calcium hydroxide paste from root canals and from the apical third. J Clin Exp Dent. 2017;9(7):e855-e860. 
22. Neuhaus KW, Liebi M, Stauffacher S, Eick S, Lussi A. Antibacterial Efficacy of a New Sonic Irrigation Device for Root Canal Disinfection. J Endod. 2016;42(12):1799-1803.

23. Plotino G, Grande NM, Mercade M, Cortese T, Staffoli S, Gambarini G, Testarelli L. Efficacy of sonic and ultrasonic irrigation devices in the removal of debris from canal irregularities in artificial root canals. J Appl Oral Sci. 2019;27:e20180045.

24. Jiang L-M, Verhaagen B, Versluis M, van der Sluis LWM. Influence of the Oscillation Direction of an Ultrasonic File on the Cleaning Efficacy of Passive Ultrasonic Irrigation. Journal of Endodontics. 2010;36(8):1372-1376.

25. Türker SA, Koçak MM, Koçak S, Sağlam BC. Comparison of calcium hydroxide removal by self-adjusting file, EndoVac, and CanalBrush agitation techniques: An in vitro study. J Conserv Dent. 2013;16(5):439-443.

26. Li D, Jiang S, Yin X, Chang JW, Ke J, Zhang C. Efficacy of Needle, Ultrasonic, and Endoactivator Irrigation and Photon-Induced Photoacoustic Streaming in Removing Calcium Hydroxide from the Main Canal and Isthmus: An In Vitro Micro-Computed Tomography and Scanning Electron Microscopy Study. Photomed Laser Surg. 2015;33(6):330-337.

27. Donnermeyer D, Wyrsch H, Burklein S, Schafer E. Removal of Calcium Hydroxide from Artificial Grooves in Straight Root Canals: Sonic Activation Using EDDY Versus Passive Ultrasonic Irrigation and XPendo Finisher. Journal of Endodontics. 2019;45(3):322-326.

28. Amaral RR, Guimarães Oliveira AG, Braga T, Reher P, de Macêdo Farias L, Magalhães PP, Ferreira PG, Ilma de Souza Côrtes M. Quantitative Assessment of the Efficacy of Two Different Single-file Systems in Reducing the Bacterial load in Oval-Shaped Canals: A Clinical Study. J Endod. 2020;46(9):1228-1234.

29. Tüfenkçi P, Yılmaz K. The Effects of Different Endodontic Access Cavity Design and Using XP-endo Finisher on the Reduction of Enterococcus faecalis in the Root Canal System. J Endod. 2020;46(3):419-424.

30. Wigler R, Dvir R, Weisman A, Matalon S, Kfir A. Efficacy of XP-endo finisher files in the removal of calcium hydroxide paste from artificial standardized grooves in the apical third of oval root canals. Int Endod J. 2017;50(7):700-705.

31. Uygun AD, Gündoğdu EC, Arslan H, Ersoy İ. Efficacy of XP-endo finisher and TRUShape 3D conforming file compared to conventional and ultrasonic irrigation in removing calcium hydroxide. Aust Endod J. 2017;43(2):89-93.

32. Keskin C, Güler DH, Sarıyılmaz E. Effect of intracanal time of triple antibiotic paste on its removal from simulated immature roots using passive ultrasonic irrigation and XP-endo Finisher. J Dent Res Dent Clin Dent Prospects. 2018;12(4):288-293.

33. Keskin C, Sariyilmaz E, Sariyilmaz Ö. Efficacy of XP-endo Finisher File in Removing Calcium Hydroxide from Simulated Internal Resorption Cavity. J Endod. 2017;43(1):126-130.

34. Sarıyılmaz E, Keskin C. Evaluation of double and triple antibiotic paste removal efficiency of various irrigation protocols. Meandros Medical and Dental Journal. 2019;20(2):129-136.

35. Lauritano, Moreo, Carinci, Della V, Di S, Sbordone, Petruzzi. Cleaning Efficacy of the XP-Endo® Finisher Instrument Compared to Other Irrigation Activation Procedures: A Systematic Review. Applied Sciences. 2019;9(23).

36. Moher D, Liberati A, Tetzlaff J, Altman DG. Preferred reporting items for systematic reviews and meta-analyses: the PRISMA statement. PLoS Med. 2009;6(7):e1000097.

37. Higgins JPT TJ, Chandler J, Cumpston M, Li T, Page MJ, Welch VA (editors). Cochrane Handbook for Systematic Reviews of Interventions version 6.0 (updated July 2019). 2019

38. Lee SJ, Wu MK, Wesselink PR. The effectiveness of syringe irrigation and ultrasonics to remove debris from simulated irregularities within prepared root canal walls. Int Endod J. 2004;37(10):672-678.

39. van der Sluis LW, Wu MK, Wesselink PR. The evaluation of removal of calcium hydroxide paste from an artificial standardized groove in the apical root canal using different irrigation methodologies. Int Endod J. 2007;40(1):52-57.

40. Sterne JAC, Savović J, Page MJ, Elbers RG, Blencowe NS, Boutron I, Cates CJ, Cheng HY, Corbett MS, Eldridge SM et al. RoB 2: a revised tool for assessing risk of bias in randomised trials. Bmj. 2019;366:14898.

41. Higgins JPT, Thompson SG, Deeks JJ, Altman DG. Measuring inconsistency in meta-analyses. Bmj. 2003;327(7414):557560.

42. DerSimonian R, Laird N. Meta-analysis in clinical trials revisited. Contemp Clin Trials. 2015;45(Pt A):139-145.

43. Kim S. Modern endodontic practice: instruments and techniques. Dent Clin North Am. 2004;48(1):1-9.

Page $15 / 22$ 
44. Stock CJ. Current status of the use of ultrasound in endodontics. Int Dent J. 1991;41(3):175-182.

45. Walmsley AD, Laird WR, Lumley PJ. Ultrasound in dentistry. Part 2--Periodontology and endodontics. J Dent. 1992;20(1):1117.

46. Jamali S, Jabbari G, Mousavi E, Ahmadizadeh H, Khorram M, Jamee A. The comparison of different irrigation systems to remove calcium hydroxide from the root canal: A systematic review and meta-analysis. Pesquisa Brasileira em Odontopediatria e Clinica Integrada. 2019;20.

47. Lauritano D, Moreo G, Carinci F, Della Vella F, Di Spirito F, Sbordone L, Petruzzi M. Cleaning Efficacy of the XP-Endo (R) Finisher Instrument Compared to Other Irrigation Activation Procedures: A Systematic Review. Applied Sciences-Basel. 2019;9(23).

48. van der Sluis LW, Versluis M, Wu MK, Wesselink PR. Passive ultrasonic irrigation of the root canal: a review of the literature. Int Endod J. 2007;40(6):415-426.

49. Yaylali IE, Kececi AD, Kaya BU. Ultrasonically Activated Irrigation to Remove Calcium Hydroxide from Apical Third of Human Root Canal System: A Systematic Review of In Vitro Studies. Journal of Endodontics. 2015;41(10):1589-1599.

50. Del Fabbro M, Corbella S, Sequeira-Byron P, Tsesis I, Rosen E, Lolato A, Taschieri S. Endodontic procedures for retreatment of periapical lesions. Cochrane Database of Systematic Reviews. 2016;(10).

51. Martin H, Cunningham W. Endosonic endodontics: the ultrasonic synergistic system. Int Dent J. 1984;34(3):198-203.

52. De-Deus G, Belladonna FG, de Siqueira Zuolo A, Perez R, Carvalho MS, Souza EM, Lopes RT, Silva E. Micro-CT comparison of $\mathrm{XP}$-endo Finisher and passive ultrasonic irrigation as final irrigation protocols on the removal of accumulated hard-tissue debris from oval shaped-canals. Clin Oral Investig. 2019;23(7):3087-3093.

53. Leoni GB, Versiani MA, Silva-Sousa YT, Bruniera JF, Pécora JD, Sousa-Neto MD. Ex vivo evaluation of four final irrigation protocols on the removal of hard-tissue debris from the mesial root canal system of mandibular first molars. Int Endod J. 2017; 50(4):398-406.

54. Xin Y, Yang J, Song KY. In vitro evaluation of the effectiveness of XP-endo Finisher file on smear layer removal after root canal instrumentation. Hua xi kou qiang yi xue za zhi [West China journal of stomatology]. 2019;37(1):48-52.

55. Kharouf N, Pedullà E, La Rosa GRM, Bukiet F, Sauro S, Haikel Y, Mancino D. In Vitro Evaluation of Different Irrigation Protocols on Intracanal Smear Layer Removal in Teeth with or without Pre-Endodontic Proximal Wall Restoration. J Clin Med. 2020;9(10).

56. Ballal NV, Gandhi P, Shenoy PA, Dummer PMH. Evaluation of various irrigation activation systems to eliminate bacteria from the root canal system: a randomized controlled single blinded trial. J Dent. 2020:103412.

57. Bao P, Shen Y, Lin J, Haapasalo M. In Vitro Efficacy of XP-endo Finisher with 2 Different Protocols on Biofilm Removal from Apical Root Canals. J Endod. 2017;43(2):321-325.

58. Sasanakul P, Ampornaramveth RS, Chivatxaranukul P. Influence of Adjuncts to Irrigation in the Disinfection of Large Root Canals. J Endod. 2019;45(3):332-337.

59. Nouran AHA, Cairo University. Effect Of XP Endo Finisher Versus Ultrasonic Activated Irrigation On Post-operative Pain In Endodontic Treatment of Symptomatic Irreversible Pulpitis. 2017. https://clinicaltrialsgov/show/NCT03096587. Accessed 22 Aug 2007.

60. Azim AA, Aksel H, Zhuang T, Mashtare T, Babu JP, Huang GT. Efficacy of 4 Irrigation Protocols in Killing Bacteria Colonized in Dentinal Tubules Examined by a Novel Confocal Laser Scanning Microscope Analysis. J Endod. 2016;42(6):928-934.

61. Rodrigues EA, Belladonna FG, De-Deus G, Silva EJ. Endodontic management of type II dens invaginatus with open apex and large periradicular lesion using the XP-endo Finisher: A case report. J Clin Exp Dent. 2018;10(10):e1040-e1044.

62. De-Deus G, Belladonna FG, Zuolo AS, Cavalcante DM, Carvalhal JCA, Simões-Carvalho M, Souza EM, Lopes RT, Silva E. XPendo Finisher $\mathrm{R}$ instrument optimizes the removal of root filling remnants in oval-shaped canals. Int Endod J. 2019;52(6):899907.

63. Silva E, Belladonna FG, Zuolo AS, Rodrigues E, Ehrhardt IC, Souza EM, De-Deus G. Effectiveness of XP-endo Finisher and XPendo Finisher R in removing root filling remnants: a micro-CT study. Int Endod J. 2018;51(1):86-91. 
64. Campello AF, Almeida BM, Franzoni MA, Alves FRF, Marceliano-Alves MF, Rocas IN, Siqueira JF, Provenzano JC. Influence of solvent and a supplementary step with a finishing instrument on filling material removal from canals connected by an isthmus. International Endodontic Journal. 2019;52(5):716-724.

65. Tavares SJO, Gomes CC, Marceliano-Alves MF, Guimarães LC, Provenzano JC, Amoroso-Silva P, Machado AG, Siqueira JF, Jr., Alves FRF. Supplementing filling material removal with XP-Endo Finisher R or R1-Clearsonic ultrasonic insert during retreatment of oval canals from contralateral teeth. Aust Endod J. 2020.

66. Marques-da-Silva B, Alberton CS, Tomazinho FSF, Gabardo MCL, Duarte MAH, Vivan RR, Baratto-Filho F. Effectiveness of five instruments when removing calcium hydroxide paste from simulated internal root resorption cavities in extracted maxillary central incisors. Int Endod J. 2020;53(3):366-375.

67. Sağlam BC, Hazar E, Koçak S, Koçak MM. Efficacy of XP-Endo Finisher and Passive Ultrasonic Irrigation on Modified Triple Antibiotic Paste Removal. Cumhuriyet Dental Journal. 2019:108-113.

68. Turkaydin D, Demir E, Basturk FB, Sazak Övecoglu H. Efficacy of XP-Endo Finisher in the Removal of Triple Antibiotic Paste from Immature Root Canals. J Endod. 2017;43(9):1528-1531.

69. Neelakantan P, Sriraman P, Gutmann JL. Removal of calcium hydroxide intracanal medicament by different irrigants and irrigating techniques: a cone beam computed tomography analysis. Gen Dent. 2017;65(6):45-49.

70. Gawdat S, Elkhodary S. Efficacy of XP-Endo finisher file compared to passive ultrasonic irrigation and conventional syringe irrigation on removal of triple antibiotic paste and calcium hydroxide. ENDO-Endod Pract Today. 2017;11(3):221-228.

71. Üstün Y, Aslan T, Sagsen B, Dincer AN. The effects of different irrigation protocols on removing calcium hydroxide from the root canals. Niger J Clin Pract. 2016;19(4):465-470.

72. Alturaiki S, Lamphon H, Edrees $H$, Ahlquist M. Efficacy of 3 different irrigation systems on removal of calcium hydroxide from the root canal: a scanning electron microscopic study. J Endod. 2015;41(1):97-101.

73. Gokturk H, Ozkocak I, Buyukgebiz F, Demir O. An in vitro evaluation of various irrigation techniques for the removal of double antibiotic paste from root canal surfaces. J Appl Oral Sci. 2016;24(6):568-574.

74. Gokturk H, Ozkocak I, Buyukgebiz F, Demir O. Effectiveness of various irrigation protocols for the removal of calcium hydroxide from artificial standardized grooves. J Appl Oral Sci. 2017;25(3):290-298.

75. Kfir A, Blau-Venezia N, Goldberger T, Abramovitz I, Wigler R. Efficacy of self-adjusting file, XP-endo finisher and passive ultrasonic irrigation on the removal of calcium hydroxide paste from an artificial standardized groove. Aust Endod J. 2018;44(1):26-31.

76. Neelakantan P, Romero M, Vera J, Daood U, Khan AU, Yan A, Cheung GSP. Biofilms in Endodontics-Current Status and Future Directions. Int J Mol Sci. 2017;18(8).

77. Kawashima N, Wadachi R, Suda H, Yeng T, Parashos P. Root canal medicaments. Int Dent J. 2009;59(1):5-11.

78. Küçükkaya Eren S, Aksel H, Parashos P. A novel model for testing the efficiency of removal of calcium hydroxide from complex root canal anatomies. Aust Endod J. 2017;43(1):5-10.

79. Tamil S, Andamuthu SA, Vaiyapuri R, Prasad AS, Jambai SS, Chittrarasu M. A Comparative Evaluation of Intracanal Calcium Hydroxide Removal with Hand File, Rotary File, and Passive Ultrasonic Irrigation: An In Vitro Study. J Pharm Bioallied Sci. 2019;11(Suppl 2):S442-s445.

80. Haupt F, Meinel M, Gunawardana A, Hülsmann M. Effectiveness of different activated irrigation techniques on debris and smear layer removal from curved root canals: a SEM evaluation. Aust Endod J. 2020;46(1):40-46.

81. Fruchi Lde C, Ordinola-Zapata R, Cavenago BC, Hungaro Duarte MA, Bueno CE, De Martin AS. Efficacy of reciprocating instruments for removing filling material in curved canals obturated with a single-cone technique: a micro-computed tomographic analysis. J Endod. 2014;40(7):1000-1004.

82. Rödig T, Döllmann S, Konietschke F, Drebenstedt S, Hülsmann M. Effectiveness of different irrigant agitation techniques on debris and smear layer removal in curved root canals: a scanning electron microscopy study. J Endod. 2010;36(12):19831987.

83. Wang Y, Guo LY, Fang HZ, Zou WL, Yang YM, Gao Y, Yang H, Hu T. An in vitro study on the efficacy of removing calcium hydroxide from curved root canal systems in root canal therapy. Int J Oral Sci. 2017;9(2):110-116.

Page $17 / 22$ 
84. Song Y, Ma JZ, Wang RY, Zhou XD, Zou L, Gao Y. Calcium hydroxide removal in curved root canals with apical transportation In Vitro. J Huazhong Univ Sci Technolog Med Sci. 2014;34(4):608-611.

85. Kenee DM, Allemang JD, Johnson JD, Hellstein J, Nichol BK. A quantitative assessment of efficacy of various calcium hydroxide removal techniques. J Endod. 2006;32(6):563-565.

86. Elnaghy AM, Mandorah A, Elsaka SE. Effectiveness of XP-endo Finisher, EndoActivator, and File agitation on debris and smear layer removal in curved root canals: a comparative study. Odontology. 2017;105(2):178-183.

87. Alves FR, Marceliano-Alves MF, Sousa JC, Silveira SB, Provenzano JC, Siqueira JF, Jr. Removal of Root Canal Fillings in Curved Canals Using Either Reciprocating Single- or Rotary Multi-instrument Systems and a Supplementary Step with the XPEndo Finisher. J Endod. 2016;42(7):1114-1119.

88. Haapasalo M, Shen Y, Wang Z, Gao Y. Irrigation in endodontics. Br Dent J. 2014;216(6):299-303.

89. Gu LS, Kim JR, Ling JQ, Choi KK, Pashley DH, Tay FR. Review of Contemporary Irrigant Agitation Techniques and Devices. Journal of Endodontics. 2009;35(6):791-804.

90. Ballal NV, Gandhi P, Shenoy PA, Dummer PMH. Evaluation of various irrigation activation systems to eliminate bacteria from the root canal system: A randomized controlled single blinded trial. J Dent. 2020;99:103412.

91. Morrison A, Polisena J, Husereau D, Moulton K, Clark M, Fiander M, Mierzwinski-Urban M, Clifford T, Hutton B, Rabb D. The effect of English-language restriction on systematic review-based meta-analyses: a systematic review of empirical studies. Int J Technol Assess Health Care. 2012;28(2):138-144.

92. Sabins RA, Johnson JD, Hellstein JW. A comparison of the cleaning efficacy of short-term sonic and ultrasonic passive irrigation after hand instrumentation in molar root canals. J Endod. 2003;29(10):674-678.

93. Sedgley CM, Nagel AC, Hall D, Applegate B. Influence of irrigant needle depth in removing bioluminescent bacteria inoculated into instrumented root canals using real-time imaging in vitro. Int Endod J. 2005;38(2):97-104.

94. Uzunoglu E, Görduysus M, Görduysus Ö. A comparison of different irrigation systems and gravitational effect on final extrusion of the irrigant. J Clin Exp Dent. 2015;7(2):e218-223.

95. Huiz Peeters H, Suardita K, Mooduto L, Gutknecht N. Extrusion of Irrigant in Open Apex Teeth with Periapical Lesions Following Laser-Activated Irrigation and Passive Ultrasonic Irrigation. Iran Endod J. 2018;13(2):169-175.

96. Boutsioukis C, Psimma Z, Kastrinakis E. The effect of flow rate and agitation technique on irrigant extrusion ex vivo. Int Endod J. 2014;47(5):487-496.

97. Amato M, Vanoni-Heineken I, Hecker H, Weiger R. Curved versus straight root canals: the benefit of activated irrigation techniques on dentin debris removal. Oral Surg Oral Med Oral Pathol Oral Radiol Endod. 2011;111(4):529-534.

98. Vaz-Garcia ES, Vieira VTL, Petitet N, Moreira EJL, Lopes HP, Elias CN, Silva E, Antunes HDS. Mechanical Properties of Anatomic Finishing Files: XP-Endo Finisher and XP-Clean. Braz Dent J. 2018;29(2):208-213.

99. Amza O, Dimitriu B, Suciu I, Bartok R, Chirila M. Etiology and Prevention of an Endodontic latrogenic Event: Instrument Fracture. J Med Life. 2020;13(3):378-381.

100. Lin CY, Lin D, He WH. Impacts of 3 Different Endodontic Access Cavity Designs on Dentin Removal and Point of Entry in 3dimensional Digital Models. J Endod. 2020;46(4):524-530.

101. Yaylali IE, Kececi AD, Ureyen Kaya B. Ultrasonically Activated Irrigation to Remove Calcium Hydroxide from Apical Third of Human Root Canal System: A Systematic Review of In Vitro Studies. J Endod. 2015;41(10):1589-1599.

102. Razumova S, Brago A, Howijieh A, Barakat H, Manvelyan A, Kozlova Y. An In Vitro Evaluation Study of the Geometric Changes of Root Canal Preparation and the Quality of Endodontic Treatment. Int J Dent. 2020;2020:8883704.

103. Vera J, Hernández EM, Romero M, Arias A, van der Sluis LW. Effect of maintaining apical patency on irrigant penetration into the apical two millimeters of large root canals: an in vivo study. J Endod. 2012;38(10):1340-1343.

104. Agarwal A, Deore RB, Rudagi K, Nanda Z, Baig MO, Fareez MA. Evaluation of Apical Vapor Lock Formation and comparative Evaluation of its Elimination using Three different Techniques: An in vitro Study. J Contemp Dent Pract. 2017;18(9):790-794.

105. Haapasalo M, Shen Y, Wang Z, Gao Y. Irrigation in endodontics. Br Dent J. 2014;216(6):299-303.

106. Chandler N, Chellappa D. Lubrication during root canal treatment. Aust Endod J. 2019;45(1):106-110. 
107. Lee NY, Kim SW, Ha SD. Synergistic effects of ultrasound and sodium hypochlorite (NaOCl) on reducing Listeria monocytogenes ATCC19118 in broth, stainless steel, and iceberg lettuce. Foodborne Pathog Dis. 2014;11(7):581-587.

108. Azimian S, Bakhtiar H, Azimi S, Esnaashari E. In vitro effect of XP-Endo finisher on the amount of residual debris and smear layer on the root canal walls. Dent Res J (Isfahan). 2019;16(3):179-184.

109. Estrela C, Costa ESR, Urban RC, Gonçalves PJ, Silva JA, Estrela CRA, Pecora JD, Peters OA. Demetallization of Enterococcus faecalis biofilm: a preliminary study. J Appl Oral Sci. 2018;26:e20170374.

110. Wilkins T, Gillies RA, Davies K. EMBASE versus MEDLINE for family medicine searches: can MEDLINE searches find the forest or a tree? Can Fam Physician. 2005;51(6):848-849.

111. Burns PB, Rohrich RJ, Chung KC. The levels of evidence and their role in evidence-based medicine. Plast Reconstr Surg. 2011;128(1):305-310.

112. Tawfik GM, Giang HTN, Ghozy S, Altibi AM, Kandil H, Le HH, Eid PS, Radwan I, Makram OM, Hien TTT et al. Protocol registration issues of systematic review and meta-analysis studies: a survey of global researchers. BMC Med Res Methodol. 2020;20(1):213.

113. Zeng X, Zhang Y, Kwong JS, Zhang C, Li S, Sun F, Niu Y, Du L. The methodological quality assessment tools for preclinical and clinical studies, systematic review and meta-analysis, and clinical practice guideline: a systematic review. J Evid Based Med. 2015;8(1):2-10.

\section{Figures}

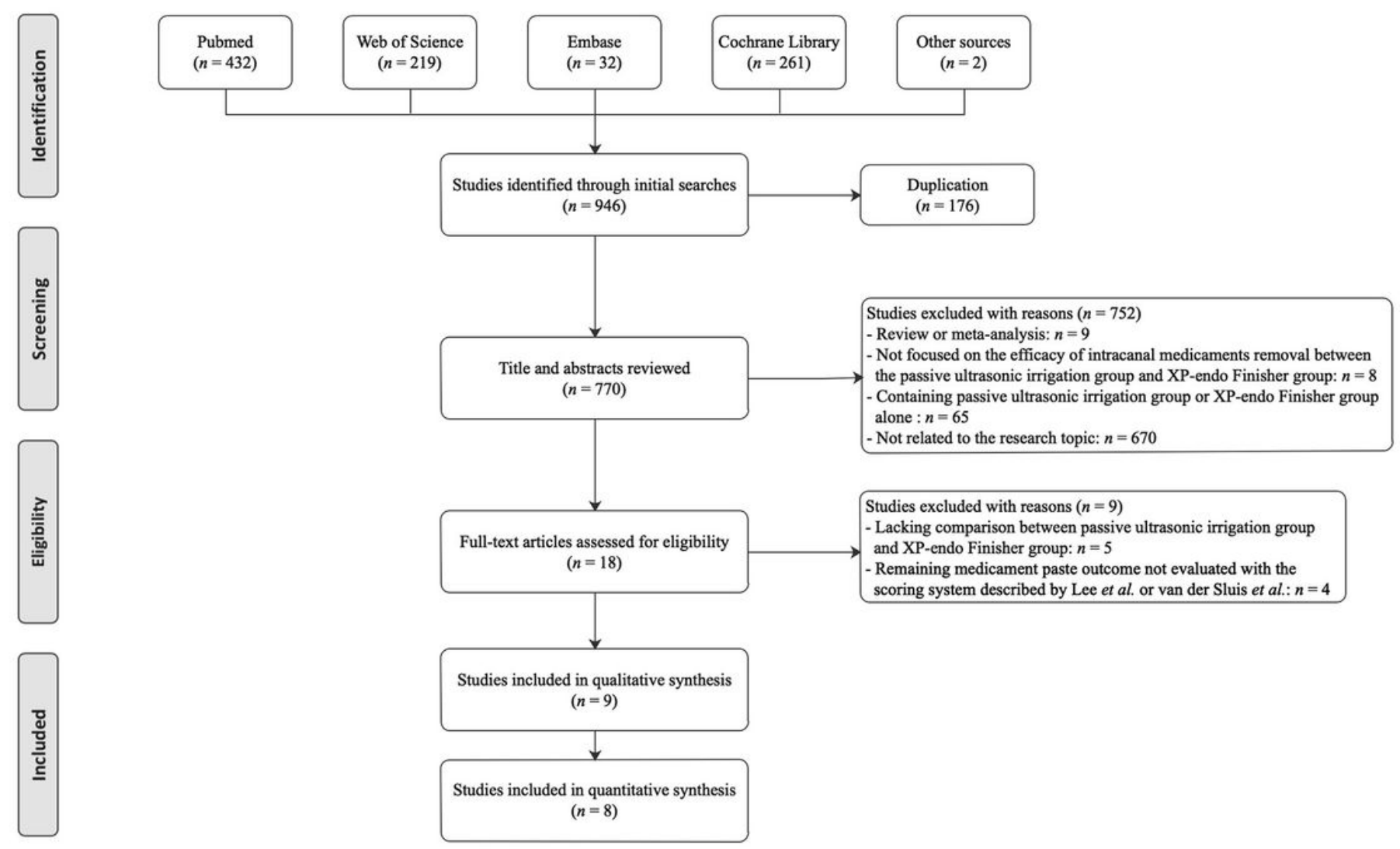

Figure 1

PRISMA flow chart of study selection process. 


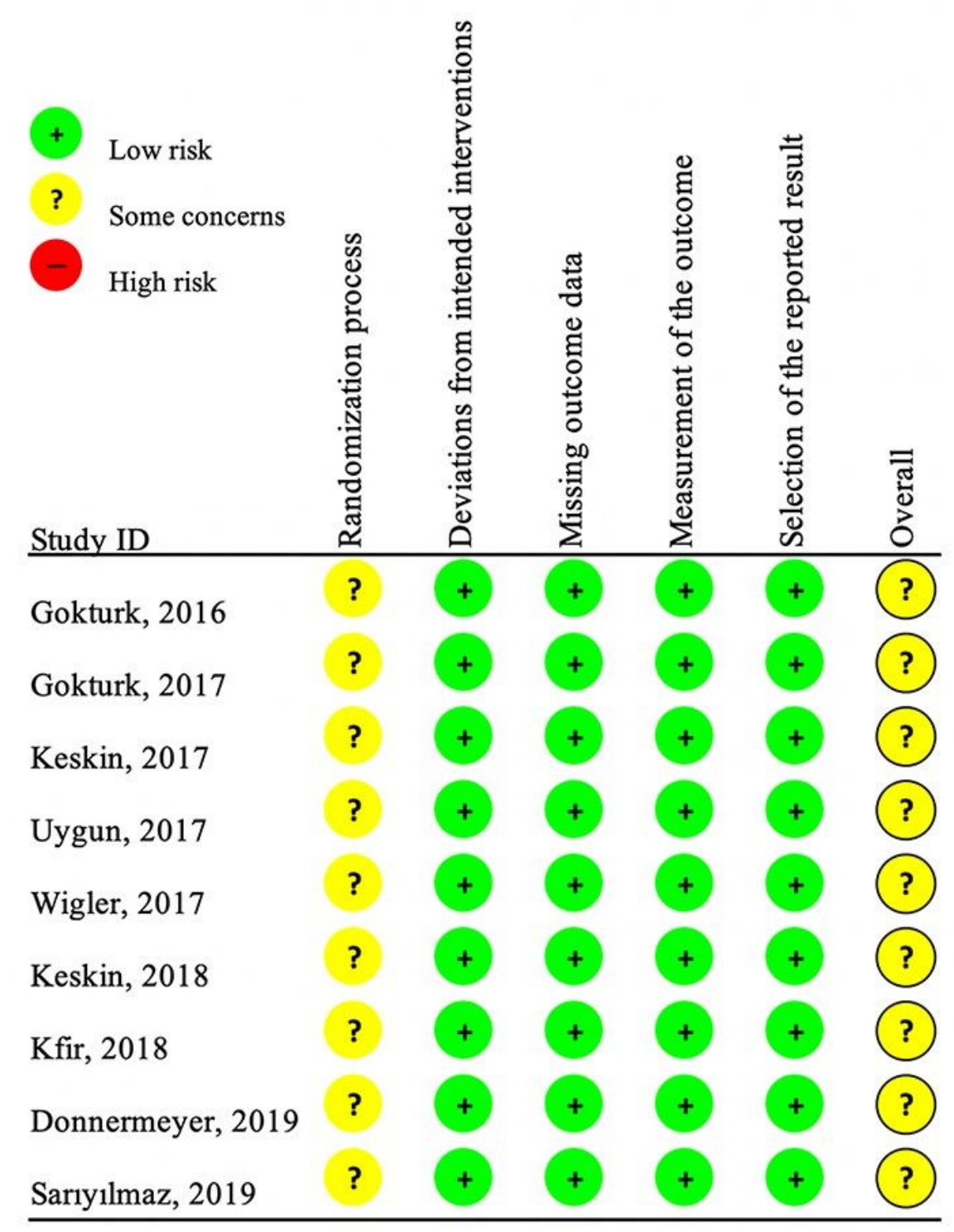

Figure 2

Risk of bias summary. 


\section{As percentage (intention-to-treat)}

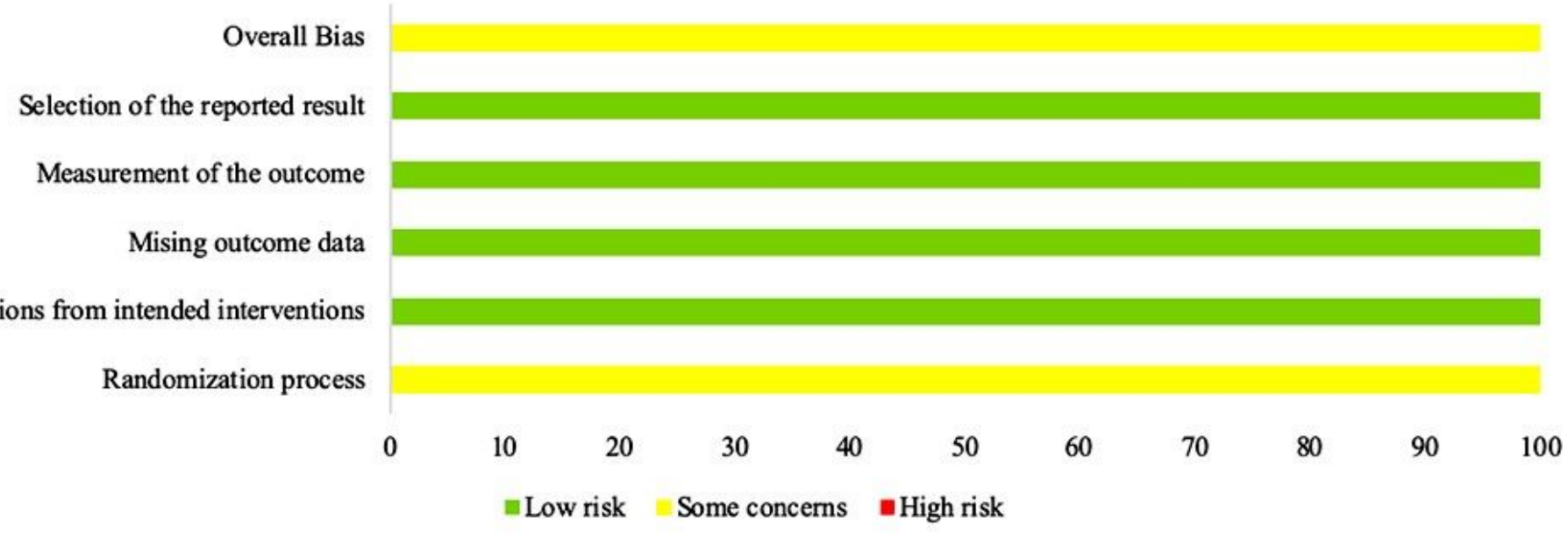

Figure 3

Risk of bias graph. Assessment is presented as percentages across all included studies.

\begin{tabular}{|c|c|c|c|c|c|}
\hline \multirow[b]{2}{*}{ Study or Subgroup } & \multicolumn{2}{|c|}{ XPF } & \multicolumn{2}{|c|}{ PUI } & \multirow[b]{2}{*}{ Weight } \\
\hline & Events & Total & Events & Total & \\
\hline Gokturk, 2016a & 10 & 15 & 5 & 15 & $7.7 \%$ \\
\hline Gokturk, 2016b & 12 & 15 & 6 & 15 & $5.5 \%$ \\
\hline Gokturk, $2016 \mathrm{c}$ & 14 & 15 & 12 & 15 & $3.7 \%$ \\
\hline Gokturk, 2017a & 11 & 15 & 3 & 15 & $3.7 \%$ \\
\hline Gokturk, 2017b & 11 & 15 & 3 & 15 & $3.7 \%$ \\
\hline Gokturk, 2017 c & 15 & 15 & 7 & 15 & $1.1 \%$ \\
\hline Keskin, 2017 & 15 & 36 & 9 & 36 & $24.2 \%$ \\
\hline Wigler, 2017 & 1 & 16 & 0 & 16 & $2.1 \%$ \\
\hline Uygun, 2017 & 18 & 20 & 17 & 20 & $7.8 \%$ \\
\hline Keskin, 2018a & 0 & 34 & 2 & 40 & $10.5 \%$ \\
\hline Keskin, 2018b & 1 & 40 & 1 & 40 & $4.5 \%$ \\
\hline Keskin, $2018 \mathrm{c}$ & 0 & 40 & 0 & 40 & \\
\hline Kfir, 2018 & 16 & 18 & 16 & 18 & $8.2 \%$ \\
\hline Sarıyılmaz, 2019a & 4 & 40 & 1 & 40 & $4.2 \%$ \\
\hline Sarıyılmaz, 2019b & 2 & 40 & 3 & 40 & $13.1 \%$ \\
\hline Total $(95 \% \mathrm{CI})$ & & 374 & & 380 & $100.0 \%$ \\
\hline Total events & 130 & & 85 & & \\
\hline
\end{tabular}

Odds Ratio

M-H, Fixed, 95\% CI

$4.00[0.88,18.26]$

$6.00[1.17,30.72]$

$3.50[0.32,38.23]$

$11.00[2.00,60.57]$

$11.00[2.00,60.57]$

$35.13[1.78,693.38]$

$2.14[0.79,5.85]$

$3.19[0.12,84.43]$

$1.59[0.24,10.70]$

$0.22[0.01,4.81]$

$1.00[0.06,16.56]$

Not estimable

$1.00[0.13,8.00]$

$4.33[0.46,40.61]$

$0.65[0.10,4.11]$

$3.09[1.96,4.86]$

Test for overall effect: $Z=4.86(P<0.00001)$

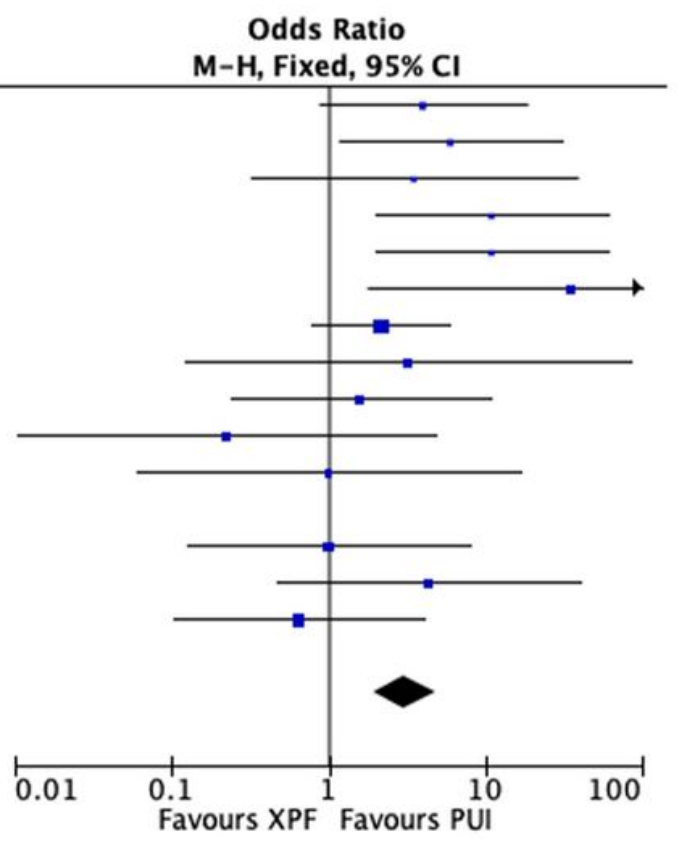

\section{Figure 4}

Forest plot and meta-analysis. Forest plots display the odds ratio related efficacy of intracanal medicament removal: XPF versus PUI. Event indicateds the number of failures. XPF, XP-Endo finisher; PUl, passive ultrasonic irrigation; $\mathrm{Cl}$, confidence interval; $\mathrm{M}-\mathrm{H}$, Mantel Haenszel test. 


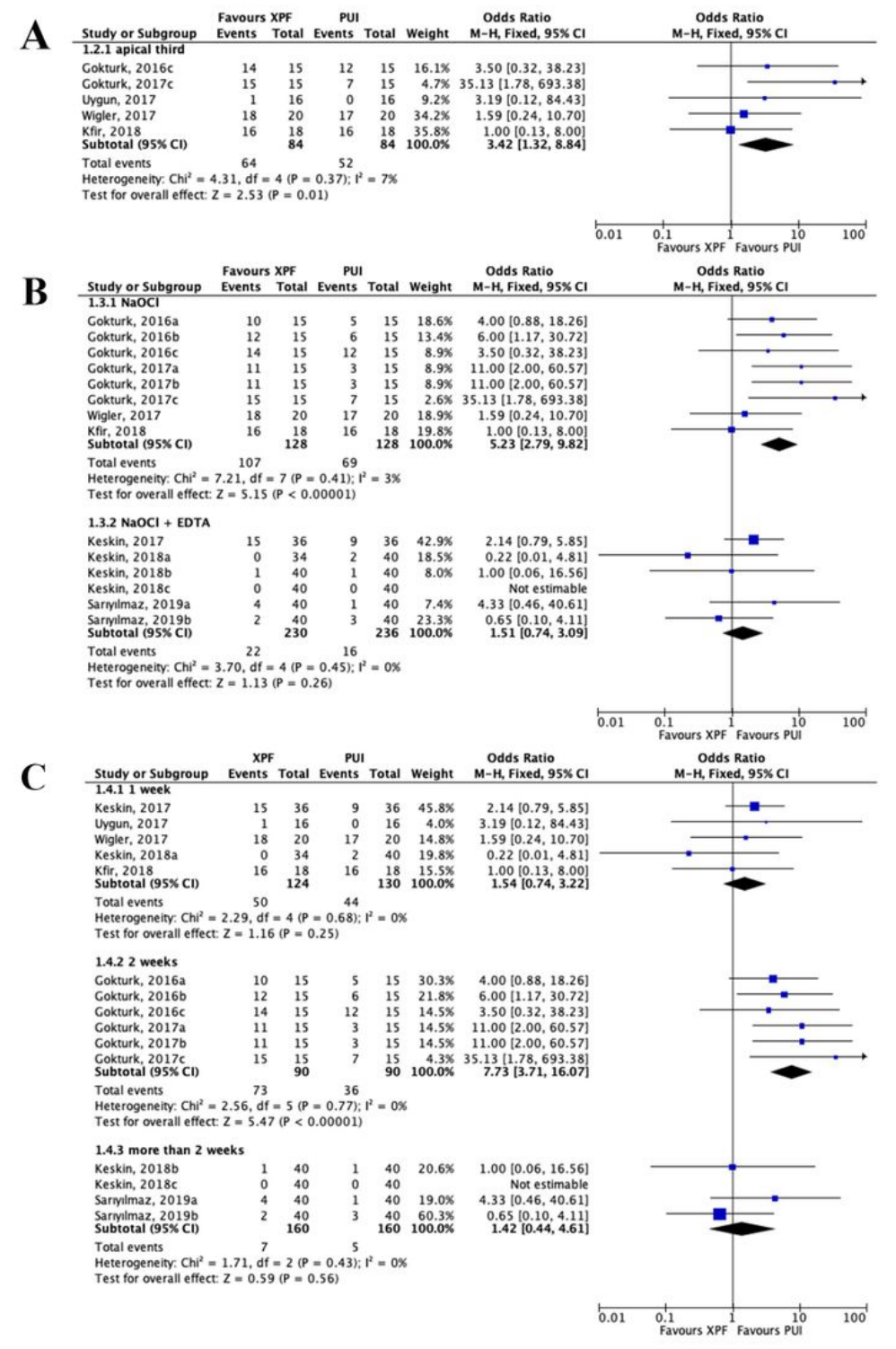

\section{Figure 5}

Forest plot of the subgroup analyses. $(A)$ anatomical areas limited to canal third; (B) irrigants type; (C) intracanal medicament time. The odds ratio depicted efficacy of intracanal medicament removal: XPF versus PUI. Event indicateds the number of failures. XPF, XP-Endo finisher; PUI, passive ultrasonic irrigation; $\mathrm{Cl}$, confidence interval; $\mathrm{M}-\mathrm{H}$, Mantel Haenszel test.

\section{Supplementary Files}

This is a list of supplementary files associated with this preprint. Click to download.

- PRISMA2009checklist.doc 